\title{
Experiencia estratégica internacional para el desarrollo profesional continuo y su impacto en la atención primaria de salud
}

\author{
Pablo Pulido, Honorio Silva, Alberto Oriol, Lewis Miller, Ada Vedilei, César Brandt, Tom Thomson, \\ Dennis Wentz, Roberto Rondón, Dave Davis, Abi Sriharan
}

Resumen. A los dinámicos y profundos cambios científicos, tecnológicos, demográficos y epidemiológicos en el área de la salud, se unen demandas de la población para mejores y más eficientes servicios. Se da el hecho de que la educación médica de pregrado y posgrado no garantiza las competencias de forma indefinida, lo que hace necesarios mecanismos que aseguren una mejor calidad asistencial. El Consorcio Proyecto Global (PGC) sugiere una estrategia integral de educación médica continua y de desarrollo profesional continuo estructurada, simplificada, evaluada y acreditada para contribuir a enfrentar estos desafíos. Se ejecutó el proyecto en dos países como prueba piloto (Rusia y Venezuela). Se implementó en cinco fases: construcción de relaciones institucionales, determinación de necesidades educativas, implementación y medición del impacto, difusión del mensaje y expansión de experiencias, y ajuste e institucionalización del proyecto. Se involucró a un número significativo de médicos que trabajan en los servicios de atención primaria de salud. Se identificaron y probaron estándares globales de educación médica continua y desarrollo profesional continuo, y se diseñó un currículo nuclear, comenzando con el área cardiovascular, con el objeto de mejorar los niveles de conocimiento y la práctica de los médicos participantes, y evidenciarlo en cambios positivos en indicadores de los pacientes usuarios. Finalmente, se llegó a la etapa de institucionalización y reforzamiento continuo, en la cual el PGC funcionó como un proyecto piloto de intercambio internacional y como posible proveedor de educación médica continua y desarrollo profesional continuo, con alta calidad, costos razonables y acreditación académica.

Palabras clave. Atención primaria. Currículo. Desarrollo profesional continuo. Educación médica continua. Estrategia de aprendizaje. Riesgo cardiovascular.

\section{An international strategic experience for continuing professional development and its impact on primary} health care

Summary. Project Globe Consortium (PGC) integrated one simplified strategy in continuing medical education and continuing professional development. It was designed as an answer to the fast and intense changes in science, technology, demography, epidemiology, the increasing demands of the population, and the changing role of physicians in the current health care systems, under the awareness that the undergraduate medical education does not guarantee the required competencies indefinitely thus implying the need of necessary mechanisms to ensure quality and professional healthcare. The project was implemented in two pilot countries (Russia and Venezuela). It was developed in five phases: building up on institutional relationships, identifying local champions; identifying educational needs; implementation and impact measurement; sharing the message and experiences; maturing adjustments and institutionalization of the project. A significant number of physicians working in primary health care settings were involved. Global standards of continuing medical education and continuing professional development were identified and tested. A core curriculum was designed, starting with cardiovascular risk factors, to improve knowledge levels and professional practice of the physicians involved, along with significant changes in patient indicators as biomarkers. Finally, the project reached the stage of institutionalization and continuous reinforcement in which the PGC represented an international networking and potential continuing medical education and continuing professional development, provider of, high quality, at reasonable costs and with academic accreditation.

Key words. Cardiovascular risk factors. Continuing medical education. Continuing professional development. Curriculum. Learning strategies. Primary health care.
Consorcio Proyecto Global, PGC (H. Silva, A. Oriol, L. Miller, T. Thomson, D. Wentz, D. Davis, A. Sriharan) Federación Panamericana de Asociaciones de Facultades y Escuelas de Medicina, FEPAFEM (P. Pulido, A. Vedilei, C. Brandt, R. Rondón) Caracas, Venezuela.

Correspondencia:

Dr. Pablo Pulido. Federación Panamericana de Asociaciones de Facultades y Escuelas de Medicina (FEPAFEM). Apartado Postal 60411. Caracas, Venezuela, 1060-A.

E-mail:

fepafempafams@ fepafempafams.org

Conflicto de intereses: No declarado.

Competing interests: None declared.

(c) 2015 FEM 


\section{Introducción}

La educación médica actual, de pregrado y posgrado, no garantiza la competencia de los profesionales en forma indefinida. El Consorcio Proyecto Global (PGC) enfocó estrategias para el aprendizaje permanente, mediante la educación médica continua (EMC) y el desarrollo profesional continuo (DPC), esenciales para los profesionales de la salud. Planteó un proyecto educativo dirigido a médicos del primer nivel de atención con la finalidad de garantizar las competencias y desempeños profesionales, el mejoramiento de los indicadores de salud y el logro de la satisfacción de los usuarios a través de una eficiente práctica profesional.

La razón de ser de este proyecto fue mejorar los servicios de salud del primer nivel de atención y mantener la actualización de las competencias profesionales de los médicos, en coherencia con la misión del PGC, que no es otra que 'promover el aprendizaje permanente, a través de la EMC y el DPC, entre los médicos generales y familiares, mediante la creación de programas educativos, estándares, recursos y alianzas, fomentando así una atención de calidad para los pacientes y sus comunidades'

\section{Procedimientos y métodos}

El proyecto se ejecutó en dos etapas: una primera fase de planificación y desarrollo, y una segunda fase de implementación y evaluación.

\section{Fase I: planificación y desarrollo}

\section{Justificación}

Idealmente, el médico debe continuar su formación a través de programas estructurados a fin de mantener los conocimientos y las competencias necesarias para prestar una atención de alta calidad.

\section{Grupos de trabajo}

Se crearon cuatro grupos de trabajo con objetivos precisos para cada uno de ellos:

- Grupo de trabajo 1. Identificó las necesidades de desarrollo profesional y educación de los médicos. Se aplicó en cada uno de los países una encuesta estandarizada en el idioma local.

- Grupo de trabajo 2. Identificó los mejores métodos de enseñanza disponibles de EMC/DPC. Realizó consideraciones de contenidos para elaborar un currículo nuclear basado en experiencias.

- Grupo de trabajo 3. Se encargó de la selección de los países a través de una matriz de conceptos y encuestas, incluyendo características nacionales. Los países debieron cumplir con los siguientes criterios: liderazgo local fuerte, enfoque geográfico, EMC/DPC local importante y red de comunicación. Fueron visitados por un representante del PGC para apreciar el cumplimiento de los criterios de selección. Los elegidos fueron Venezuela, Colombia, Turquía y Rusia (Fig. 1).

- Grupo de trabajo 4. Se encargó de redactar misión, visión, metas y objetivos del PGC.

\section{Alianzas}

En una fase posterior del PGC, se invitó a organizaciones e instituciones, así como a individuos claves, a unirse al comité directivo inicial.

\section{Recomendaciones preliminares}

Se seleccionó un método de acreditación de EMC/ DPC compatible con un objetivo de reciprocidad de créditos de manera internacional.

La financiación del PGC en esta fase de planificación provino de fundaciones, asociaciones y sociedades científicas, así como de particulares. Adicionalmente, y para la ejecución de todo el proyecto, se contó con el apoyo económico básico del Pfizer Institute for Medical Humanities.

\section{Fase II: implementación y evaluación}

Se desarrolló en cinco etapas (Fig. 2).

\section{Construcción de relaciones institucionales}

Para comprender el país y desarrollar el equipo local de líderes del proyecto, se establecieron relaciones con educadores médicos, academias e instituciones de salud de los países, lo que implicó conocer sus necesidades de EMC y DPC, así como aumentar su potencial. Se identificó el liderazgo conductor, con el cual se firmó un 'memorándum de entendimiento', y posteriormente se conformaron grupos locales, se confeccionó el cronograma de ejecución y se elaboró el presupuesto.

\section{Determinación de necesidades educativas}

El PGC trabajó con los países en la identificación de necesidades educativas. El análisis de las necesidades de aprendizajes se hizo a nivel individual, organizacional y del sistema de salud. Con los resultados obtenidos se diseñó el currículo básico, enfocado en las áreas prioritarias detectadas.

Las técnicas utilizadas para detectar las necesidades de capacitación incluyeron encuestas en línea de 20 preguntas aplicadas a un grupo significativo de 
médicos parte de los servicios de atención primaria de salud, con la finalidad de detectar sus áreas prioritarias de capacitación, así como sus preferencias en relación con la forma de recibir dicho entrenamiento. Estas encuestas se aplicaron en los países seleccionados para realizar el estudio: Colombia, Rusia, Turquía y Venezuela.

Se analizaron los índices de morbimortalidad de cada uno de estos países. También se utilizaron reuniones de tipo grupo focal, en cada uno de los países seleccionados, con representantes de cada una de las instituciones participantes en la educación de médicos, como facultades de medicina, sociedades científicas, instituciones gremiales, ministerios de salud e industria farmacéutica.

\section{Implementación y medición del impacto}

El PGC ofreció a cada país la oportunidad de participar en un estudio para evaluar la eficacia de la EMC/DPC para cambiar favorablemente la conducta de los médicos y los indicadores de salud de los pacientes.

El PGC utilizó una infraestructura técnica y LMS (Learning Medical System) para almacenar el software, y proveyó servicios de mantenimiento y de registro, en especial sobre las licencias de los softwares de uso pedagógico.

\section{Difundir el mensaje y expandir la experiencia}

Consistió en la presentación y publicación de los resultados de los estudios pilotos y su discusión en simposios y conferencias internacionales, y en la discusión de un plan de crecimiento para extender el impacto del PGC hacia otros países.

\section{Ajuste e institucionalización del proyecto}

El PGC funcionó como un centro de intercambio de información internacional y como un proveedor de EMC/DPC de alta calidad, con costos razonables. El trabajo de campo se realizó conjuntamente con las asociaciones médicas nacionales mediante el otorgamiento de créditos académicos y otras acreditaciones.

\section{Resultados}

Se ejecutó el proyecto en dos países como prueba piloto, Rusia y Venezuela. Se puso en práctica en cinco fases, se involucró a un número significativo de médicos que trabajan en servicios de atención primaria y, basado en ese resultado, se diseñó un currículo nuclear, comenzando con el área de riesgo cardiovascular, para mejorar los conocimientos
Figura 1. Estrategias en proyectos.

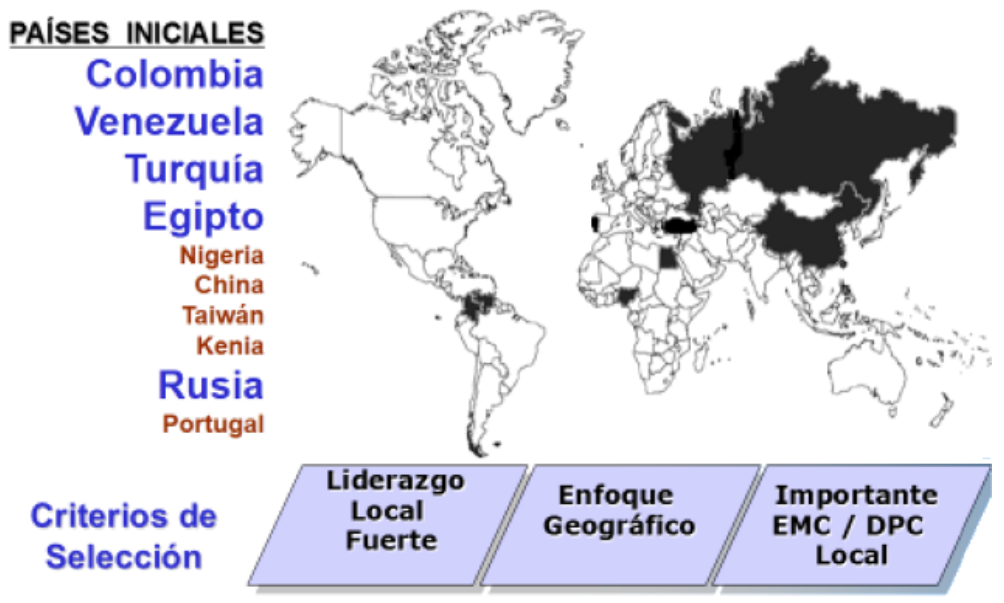

Figura 2. Implementación del Consorcio Proyecto Global.

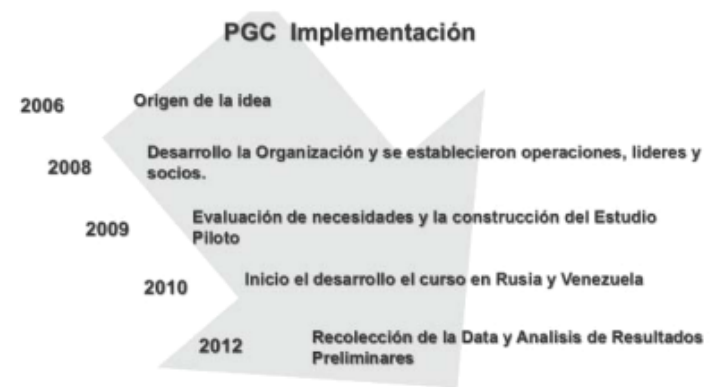

y la práctica profesional de los médicos participantes, todo ello evidenciado en los cambios positivos en los indicadores-biomarcadores de los pacientes.

En base a las encuestas, la participación total fue del $40 \%$ de médicos familiares y del $60 \%$ de médicos generales. Por otra parte, el $55 \%$ de los encuestados había participado en actividades educativas estructuradas de más de 50 horas durante el último año, mientras que el $3 \%$ de los encuestados no había participado en ninguna actividad. También se apreció que los médicos encuestados tenían preferencia por cursos dictados en el idioma local, que contaran con aval académico, y con modalidad de participación combinada entre presencial y virtual. Hay que resaltar que las patologías más frecuentes en sus consultas eran las enfermedades car- 
Tabla. Módulos de factores de riesgo cardiovascular.

\begin{tabular}{lll}
\hline & Objetivos generales & Temas \\
\hline $\begin{array}{l}\text { Módulo I. Características } \\
\text { generales de los factores } \\
\text { de riesgo cardiovascular }\end{array}$ & $\begin{array}{l}\text { Ofrecer el conocimiento necesario para identificar y } \\
\text { diagnosticar los diferentes síndromes, patologías y estilos de } \\
\text { vida que son considerados factores de riesgo cardiovascular }\end{array}$ & $\begin{array}{l}\text { Riesgos cardiovasculares } \\
\text { Factores clásicos de riesgo cardiovascular } \\
\text { Otros factores de riesgo }\end{array}$ \\
\hline $\begin{array}{l}\text { Módulo II. Manejo clínico } \\
\text { de pacientes con factores }\end{array}$ & $\begin{array}{l}\text { Proveer el conocimiento necesario para capacitar apropiadamente } \\
\text { en el manejo de pacientes con factores de riesgo cardiovascular } \\
\text { y hacer seguimiento de las recomendaciones }\end{array}$ & Manejo terapéutico \\
\hline $\begin{array}{l}\text { Módulo III. Cambios conducto y control } \\
\text { en los pacientes con factores } \\
\text { de riesgo cardiovascular }\end{array}$ & $\begin{array}{l}\text { Proveer el conocimiento necesario para el desarrollo de } \\
\text { capacidades y destrezas para promover modificaciones } \\
\text { de los estilos de vida en sus pacientes }\end{array}$ & Educación terapéutica \\
\hline
\end{tabular}

diovasculares, respiratorias, parasitarias y digestivas, mientras que las patologías en las que prioritariamente necesitaban capacitación eran enfermedades cardiovasculares, dermatológicas, digestivas y neoplásicas.

El objetivo general fue ofrecer a los médicos de atención primaria una experiencia de educación formal para el manejo adecuado de los riesgos cardiovasculares en la práctica cotidiana y así mejorar los resultados de la atención a los pacientes, midiendo la efectividad de la intervención educativa por medio de la adquisición de competencias en su práctica clínica y el refuerzo de los resultados en la mejora de sus pacientes.

Además del conocimiento sobre los riesgos cardiovasculares, se incluyó el manejo de temas básicos, como la relación médico-paciente, la educación del paciente y estrategias para modificar el estilo de vida del paciente.

Para el desarrollo de un currículo nuclear, se realizaron discusiones para la elaboración de los protocolos, con la asesoría de grupos internacionales, basados en las necesidades detectadas en las encuestas realizadas, y con prioridades referidas a enfermedades infecciosas, respiratorias, cardiovasculares, metabólicas, osteoarticulares, ginecoobstétricas, geriátricas, pediátricas y cáncer.

En las distintas encuestas realizadas se demostró la falta de preparación de los médicos familiares y generales en relación con el manejo de los riesgos de las enfermedades cardiovasculares, de donde se deduce la inmediata necesidad de realizar programas de EMC/DPC para satisfacer esta demanda, metodológicamente mediante cursos combinados tanto presenciales como en línea.

El curso nuclear en riesgo cardiovascular implementado constó de tres módulos, con un total de seis temas interrelacionados e integrados entre sí, ofrecidos en línea, de forma asincrónica, además de tres sesiones presenciales tipo talleres, de cuatro horas de duración cada uno (Tabla). Se utilizó la estrategia del aprendizaje basado en problemas. En los talleres se intercambiaron experiencias y se reforzó el desarrollo de aspectos que mejoran la conducta y la actitud en la práctica médica. Se realizaron actividades tutoriales, foros e intercambio de opiniones, todo en línea, para facilitar la interrelación entre estudiantes y profesores.

Los médicos que participaron en servicios de atención primaria tuvieron seguimiento durante las 14 semanas, con una comunicación directa y abierta entre estudiantes, profesores y tutores. Los pacientes fueron seleccionados y monitorizados por sus propios médicos, y además fueron evaluados periódicamente en los indicadores de factores de riesgo cardiovascular.

\section{Conclusiones}

El PGC, ente de carácter académico, sin fines de lucro y dedicado a promover un compromiso y una excelente práctica profesional, se inicia con la identificación de la organización de los servicios de salud, las deficiencias de la formación de los médicos en atención primaria, las propuestas educacionales necesarias y convenientes para mejorar las competencias y la conducta terapéutica de los médicos, así como la adhesión de los pacientes a su respectivo tratamiento.

El PGC, como alternativa de acción práctica, logró que se desarrollaran relaciones de asociación y compromiso con instituciones que prestan atención primaria de salud, incluyendo las de financiación fiscal pública, educadores médicos y academias para compartir la filosofía del proyecto, realizar encues- 
tas de necesidades y poner en práctica actividades de EMC/DPC mediante protocolos educativos, usando metodologías combinadas, en línea y presenciales, de autoeducación y autoevaluación, y de evaluación externa, lo que lleva a cambios en las competencias y en la conducta, tanto de los médicos como de los pacientes, aspectos que se midieron como parte esencial del proyecto. También implicó la elaboración y desarrollo de un currículo nuclear, con asesoría internacional para responder al reto.

El curso sobre factores de riesgos cardiovasculares demostró cómo, en términos de EMC/DPC, se obtuvieron mejoras en las competencias y en el comportamiento de los pacientes con respecto a sus respectivas patologías. Al finalizar el curso, se publicaron los resultados de los estudios pilotos y se hizo una evaluación de las necesidades del país con los autores locales como contribuyentes, se presentaron los resultados en simposios y conferencias internacionales, y finalmente se trabaja en un plan de crecimiento para extender el impacto del PGC a otros países.

\section{Bibliografía}

1. World Federation for Medical Education. Estándares globales de la WFME para la mejora de la calidad. Educ Med 2004; 7 (Supl 2): S39-52.

2. Pulido P, Rondón R. Venezuelan medical education. In McGuire CH, Veach RM, Aziz LR, Linda K, eds. International handbook of medical education. London: Greenwood; 1994.

3. Davis D. Does CME work? An analysis of the effect of educational activities on physician performance on health care outcomes. Int J Psychiatry Med 1998; 28: 21-39.

4. Fordis M, King JE. Comparison of the instructional efficacy of internet based CME with live interactive CME workshops. JAMA 2005, 294: 1043-51.

5. Bloom BS. Effects of continuing medical education on improving physician clinical care and patient health a review of systematic reviews. Int J Technol Assess Health Care 2005; 21: 380-5.

6. Mansouri M, Lockyer J. A metaanalysis for continuing medical education effectiveness. J Contin Educ Health Profes 2007; 27: 6-15.

7. Marinopoulos SS, Dorman T, Ratanawongsa N. Effectiveness of continuing medical education. Evidence Report/Technology Assessment no. 147. Rockville, MD: Agency of Healthcare Research \& Quality; 2007.

8. The ACCME's essential areas and their element. Accreditation Council for Continuing Medical Education. URL: http://www. acme.org. [18.10.2011].

9. Frenk J, Chen L, Bhutta Z. Health professionals for a new century: transforming education to strengthen health systems in an interdependent world. Lancet 2010; 376: 1923-58.

10. Miller L, Jain S, Moyland S, Pulido P. Continuing medical education for the world; spreading to Latin America, Asia, Africa and the Middle East. In Wentz D, ed. Continuing medical education, looking back, planning ahead. Lebanon, NH: Dartmouth University Press; 2011. p. 347-56.

11. Pulido P, Cravioto A, Pereda A. Changes, trends and challenges of medical education in Latin America. Med Teach 2006; 28 : 1: 24-9.

12. Vartanyan F, Silva H, Avtandilov AG, Borisov KN, Shurandina IS, Pukhaeva AA, et al. Efectos de una intervención educativa en el manejo clínico de los factores de riesgo cardiovascular en atención primaria de salud. Experiencia con Project Globe Consortium en Rusia. FEM 2013; 16: 131-6.

13. Kirkpatric DL, Kirkpatrick JD. Evaluating training programs. 3 ed. San Francisco, CA: Berrett-Koehler Publishers; 2006.

14. Pulido P, Silva H, Vedilei A, Brand C. Management of cardiovascular risk in Venezuela by educating physicians working in primary healthcare services. [En proceso de publicación.] 